latitudes where the lines of force come down towards the Earth and converge the particles are turned back or "mirrored". Thus they are trapped in stable helical paths with repeated mirroring in alternate hemispheres. A line of force of the Earth's magnetic field is described by its distance from the Earth's centre at the highest point, that is, where it crosses the equator. This distance is written $L R_{\mathrm{e}}$ where $R_{\mathrm{e}}$ is the Earth's radius, and we speak of the $L$ value of a line of force. The radiation belts extend up to about $L=7$ or 8 with two maxima of intensity around $L \sim 1 \frac{1}{2}$ and $L \sim 3 \frac{1}{2}$. In between there is a minimum or "slot", in the range $2<L<3$, which was shown in Van Allen's first map of the radiation belts (Van Allen and Frank, Nature, 183, 430; 1959).

The particles of the radiation belts can sometimes generate electromagnetic waves of audio frequency. These travel in the whistler mode to the ground and are observed as the remarkable rising and falling whistles that Storey ( $o p$ cit.) called the 'dawn chorus'. The particles can give amplification of whistler waves, operating in a similar way to a travelling wave tube amplifier and this is the amplification needed to explain the observed strengths of some whistlers. Emission of radiation from the particles can be triggered by electromagnetic waves and triggering by whistlers and by signals from radio transmitters of very low frequency has been known for many years. Triggering by the harmonics of the $60 \mathrm{~Hz}$ electric power system of North America has been observed more recently (Helliwell, Katsufrakis, Bell and Raghuram, J. geophys. Res., 80, 4249; 1975). Observations were made simultaneously at Siple in Antarctica and at Robertval, Quebec which is near the aluminium smelting plant at Arvida which uses roughly $1,000 \mathrm{MW}$ at $60 \mathrm{~Hz}$. The rectifiers generate high harmonics, up to about the 90th, that is about $5 \mathrm{kHz}$, which enter the magnetosphere and trigger emission at other frequencies. The triggering is believed to occur mainly over the equator. The triggered emissions travel to Earth in the whistler mode and can be observed at Siple.

Electromagnetic waves can, in turn, influence the particles of radiation belts so as to change their orbits. One possibility is that the pitch angle of a particle's path is decreased, so that it is made to move more nearly parallel to its guiding line of force. This means that its helical path is more open and it can penetrate further down towards the ground. Then, for many of the particles, mirroring does not occur. They are "precipitated" into the Earth's lower atmosphere, and the radiation belts are correspondingly depleted. Dungey (Planet. Space Sci., 11,
591 ; 1963) suggested that electromagnetic signals from the Earth might modify the radiation belts in this way, and there was speculation as to whether the electron slot in the range $2<L<3$ could be explained by this mechanism.

A recent study of emissions, mainly in the audio frequency range, made with the satellites Ariel 3 and 4, has greatly strengthened this possibility (see Bullough, Tatnall and Denby, page 401 in this week's issue of Nature). The analysis shows that harmonics of the 60 $\mathrm{Hz}$ power system in North America might contribute appreciably to the precipitation of particles. VLF radio transmissions from NAA (Maine, 17.8 $\mathrm{kHz}$ ) and GBR (Rugby, $16 \mathrm{kHz}$ ) may also play a part. But thunderstorms in the American and African continents deliver a very large amount of electromagnetic energy into the magnetosphere, and further observations and analysis will be needed before we can be certain that the man-made sources of energy are really significant.

\section{The importance of being rotavirus}

from June D. Almeida and Arie J. Zuckerman

LATELy there has been considerable interest in the characterisation by direct electron microscopy of viruses in faeces (see Nature, 254, 557; 1975). Recent publications report reoviruslike agents in stools (Kapikian et al., Science, 185, 1049; 1974), infantile gastroenteritis virus or IGV (Newman et al., Nature, 258, 631; 1975), duovirus (Bishop et. al., Lancet, ii, 1281; 1973), rotavirus and orbivirus (Flewett et al., J. clin. Path., 27, 603; 1974). To the uninitiated it might seem that there has been a vast increase in the number of virus types to be identified in acute gastroenteritis. Closer examination reveals, however, that each of these descriptions concerns the same virus, which is now associated with acute diarrhoea in the human infant, the calf, the mouse, the pig and the foal. The outstanding feature of this virus is its morphology and at least three of the terms suggested are based on this morphology. Duovirus refers to the double-shell construction, orbivirus to its overafl circular appearance and rotavirus refers to the spoke-like outer projections which give it a distinctive wheel-like appearance. It now seems that under certain circumstances the same virus can reveal a distinctive starlike pattern which is shown in the figure and interpreted in the inset diagram. The structure illustrated was

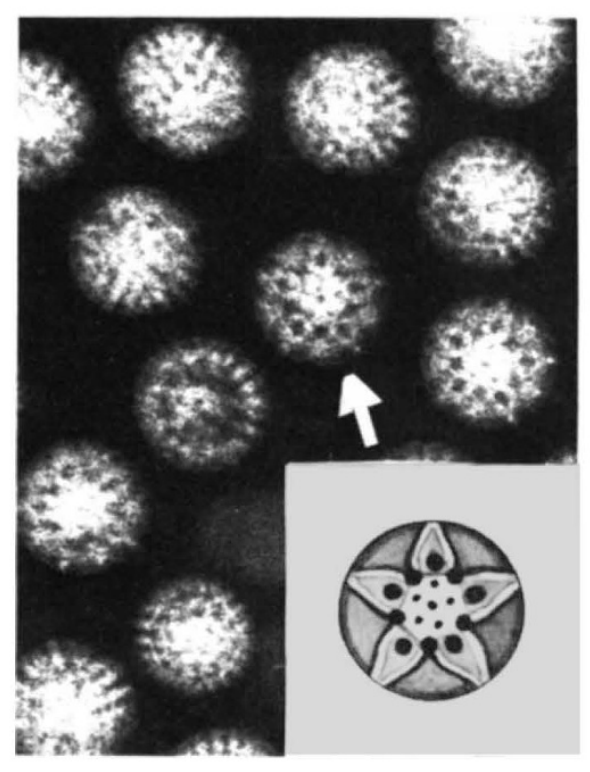

A group of rotavirus particles from a calf faecal homogenate. The majority show the morphology associated with this virus type and both complete and incomplete forms are present. In addition there are two particles showing the star arrangement described. $\times 225,000$. Inset, diagrammatic representation of the stellate particles based on the detail present in a number of them. To be compared with the arrowed particle in the main micrograph.

obtained from a specimen of faeces from a calf with diarrhoea. The titre of the particles was particularly high, estimated as being in the order of $10^{11}$ particles $\mathrm{ml}^{-1}$. Rotaviruses consist of two concentric capsids each built up from distinctive, geometrically arranged subunits. The star-like morphology demonstrated may be a superimposition pattern formed by the interaction of these two structures. On the other hand, it may be that the stellate form shown in the figure is the intact form of this virus, and the ability to distinguish two components implies that breakdown has occurred.

This finding suggests that the situation could be further confused by the name astrovirus, one already used for gastroenteritis viruses by Madeley and Cosgrove (Lancet, ii, 451; 1975) or even stellavirus. But rather than introduce yet another name for this already terminologically overladen group it seems better to fit this new morphological finding to an existing term. The diagramatic inset emphasises a certain similarity between the new morphological feature of this virus group and a mediaeval "wheel of fortune". Thus two separate aspects of this virus group exhibit a wheel-like appearance, in turn suggesting that the term rotavirus may well be the most appropriate for this group which seems to move in wide circles throughout the animal kingdom. 